\title{
Re-using archived qualitative data - where, how, why?
}

\section{Louise Corti}

Received: 9 April 2005/ Accepted: 10 November 2005

(C) Springer Science+Business Media B.V. 2006

Abstract "Qualitative data" are the central issue of this article. Qualitative data are a particular category of data within the social sciences, where data have been predominantly of a quantitative nature. Qualitative data could enrich social science research in many ways. The re-use of this particular group of data is however a new challenge for social science data archives. A new methodology has to be developed when dealing with these data, based on a combination of social science methodology and traditional archival descriptions. An additional question discussed in the article is what the best place should be for archiving and disseminating qualitative data: in research (social science) data archives or in the more traditional libraries and archives?

12 Keywords Archives $\cdot$ Data $\cdot$ Research data $\cdot$ Qualitative data Data archives

13 ESDS - UKDA - Secondary analysis of data $\cdot$ Methodology $\cdot$ Data provision ·

14 Social science

\section{Introduction}

Archived qualitative data are a rich and unique, yet too often unexploited, source of research material. Qualitative data are collected across a range of social science disciplines and typically aim to capture lived experiences of the social world and the meanings people give these experiences from their own perspectives. They offer information that can be reanalysed, reworked, and compared with contemporary data. In time, too, archived research materials can prove to be a significant part of our cultural heritage and become resources for historical as well as contemporary research. While there is a well-established tradition in social science of reanalysing

L. Corti $(\triangle)$

ESDS (Economic and Social Data Service) Qualidata,

Outreach and Training, UK Data Archive at the University of Essex,

University of Essex, Colchester CO4 3SQ, UK

e-mail: corti@essex.ac.uk

\begin{tabular}{|l|lll|}
\hline & & & $\underline{\underline{C}}$ Springer \\
& Journal : 10502 & Dispatch : 14-12-2006 & Pages : 20 \\
Article No. : 9038 & $\square$ LE Code : ARCS633 & $\square$ CP & $\square$ TYPESET \\
\hline
\end{tabular}


quantitative data, there is not yet a well-developed paradigm, nor an evident blossoming research culture, for secondary qualitative data analysis. The lack of discussion in the current literature on the benefits and limitations of such approaches is evident. This paper discusses some of the methodological, ethical and theoretical considerations relating to the secondary analysis of qualitative data.

The second part of this paper addresses more pragmatic issues involved in the acquisition, preservation, dissemination of and support for qualitative data. An overview of some of the key challenges faced by an archive for acquiring and processing qualitative data collections will be provided. Where best do qualitative data collections sit? In traditional libraries or paper archives alongside historical documents, or as part of more holistic digital collections of contemporary social science research resources? This question relates to data accessibility and dissemination, cataloguing methods, user documentation and finding aids, and outreach and training strategies that promote the use of qualitative data archives. ESDS (Economic and Social Data Service) Qualidata will be used as a case study to contextualize this debate.

\section{Kinds of qualitative data available for secondary analysis}

Often a diversity of methods and tools rather than a single one are encompassed. The types of data collected vary with the aims of the study and the nature of the sample. Samples are most often small, but may rise to 500 or more informants. Such data include interviews-whether in-depth or unstructured, individual or group discussion-fieldwork diaries and observation notes, structured and unstructured diaries, personal documents, or photographs. For the sake of a simple definition of 'qualitative' any research material that is collected from studying people can be included, unless it has been transformed into numerical values (e.g. in a spread sheet, database or statistical software), in which case it becomes quantitative.

Thus any one study may yield a wide range of data types for archiving. Moreover most of these types of data may be created in a variety of formats: digital, paper (typed and hand-written), audio, video and photographic. But, increasingly data are now "born digital" data in the sense that the text is word-processed, and audio recordings are collected and stored as MP3 files.

In Britain, the 1950s onwards saw an unprecedented growth of quantitative and qualitative social research resulting in the spread of its themes and the development of its methods. From the 1960s into the 1970s, sociology was not only an exceptionally popular subject with students, but was also given more national research resources than at any time before or since. This enabled social researchers to carry out studies of a scale unlikely ever to be equalled. An example is Peter Townsend's in-depth investigation into the nature and status of older people's institutions in post-war Britain. The publication resulting from this research, The Last Refuge (Townsend 1962) was considered a pioneering piece of research when it was published in 1957 and attracted much publicity for its focus on an important and hitherto neglected area of policy, and also for its methodology and its policy recommendations. But Peter Townsend's meticulously preserved fieldwork descriptions of old people's institutions and accompanying interviews, now archived and available to researchers at the University of Essex, are equally significant. Not only do they provide a rich descriptive context of policy at that time, but they also represent a 
glimpse behind the final polished policy reports-exposing how the researcher approached the study and the methods he used.

Clearly the scope and format of data usually determine its potential for secondary analysis. For example, data from a research study that collected, recorded and transcribed a hundred or so in-depth interviews and documented detailed field notes, particularly when based on a clear sampling strategy, are much more likely to be useful than a small focussed set of interview notes from twenty or so brief semistructured interviews. These have more limited re-use value as the final publications will usually have 'mined' the limited data quite thoroughly, offering less new use value. But as with many archived sources, sometimes the most exciting discoveries arise from re-examining material which hitherto has not been thought worth researchers' attention. David Zeitlyn argues that field photographs and audio-visual material are probably among the most prolific and least exploited resources produced by anthropologists (Zeitlyn 2000).

\section{The availability of qualitative data for re-use}

85

86

87

88

89

90

91

92

93

94

95

96

97

98

99

100

101

102

103

104

105

106

107

108

109

110

111

112

113

114

115

If we take a look across the world in an attempt to identify qualitative data sources that could be openly consulted, we immediately encounter problems. The first is the absence in most countries of any national effort to either gather together or draw attention to existing research sources. The second is the lack of infrastructures and also of agreed practical procedures for preparing, storing and disseminating qualitative data.

Throughout the world there are innumerable archives which collect (mainly historical) qualitative material, as well as a large number of sound archives and ethnographic archives, but there are few common descriptive standards, access to many collections is poor, and there are no integrated resource discovery tools. For example, while university repositories may hold a Professor's research interviews from past investigations, they are often buried away in the boxes of personal papers, and the hand-lists typically provided by such archives are not at all conducive to locating the raw data. Moreover, many are still not digitised. Corti and Thompson (2004) describe the general availability of qualitative data sources around the world, and discuss local and national local archival initiatives arising over the past decade.

One of the earliest and perhaps best known sources in Britain is the collection of papers resulting from the 1930s social research organisation, Mass-Observation. These were established as a notably well-organised and accessible public archive at the University of Sussex in the early 1970s, and since then have attracted a significant number of researchers (Sheridan 2000). More typically, other data collections that were retained were stored as in-house research resources, such as the Berkeley and Oakland cohorts collected from the 1920s to the 1990s at the Institute for Human Development at Berkeley. It has also been not unusual for the papers of eminent scholars, sometimes representing a lifetime's research, to be transferred on retirement to their local university archives. Such papers may include not only primary research data, but also administrative documents about the research, such as grant proposals and correspondence. A collection may also contain 'secondary' sources utilised for a particular research study, such as newspaper clippings, organisational or medical records. But, more generally, attempts to archive qualitative research material were both rare and unsystematic. Since the 1990s new technologies have led

\begin{tabular}{|l|llll|} 
& & & & \\
\hline
\end{tabular}


116 to new possibilities for sharing qualitative data, especially through online resources

117 and databases.

\section{National archiving}

119 In Britain, as indeed in most western countries, until recently no infrastructure existed for the systematic archiving and dissemination of qualitative data from social science research. The UK's Economic and Social Research Council (ESRC; then SSRC) had already recognized from very early on in 1967, the value in retaining the most significant machine-readable data from the empirical research which it funded by establishing a national Data Archive. Since the 1970s, national social science data archives have been formed across the world acquiring a significant range of data relating to society, both historical and contemporary, from sources including surveys, censuses, registers and aggregate statistics. These have become centres of expertise in acquiring, describing, sharing, providing access to and preserving data. International networks of data services have been established for the social sciences which foster co-operation on key archival strategies, procedures and technologies.

Thus crucial survey data can be re-analysed by other researchers, and the money spent on research has become not only an immediate outlay but an investment for the future. There was, however, a significant gap in this policy in that qualitative data were rarely acquired, even when much interview data became transcribed in word processed form. This was largely because non-numerical data were simply not viewed as 'data'. Nor was there any willingness on the researchers' part to share their qualitative data. When a small pilot study commissioned by the ESRC was carried out by Paul Thompson in 1991 (Thompson 1991), it was revealed that $90 \%$ of qualitative research data was either already lost, or at risk, in researchers' homes or offices. However the ten percent 'archived' were found not to have the basic requirements of an archive, such as physical security, public access, reasonable catalogues, with recorded material or listening facilities. It was further calculated that it would have cost at least $£ 20$ million to create a resource on the scale of that at risk. For the older British sociological material, moreover, the risk was acute, and the need for action especially urgent. This was borne out by the very recent destruction of research data on the classic British community studies of Banbury (Stacey 1974); on race and conflict in Sparkbrook (Rex and Moore 1967).

In 1994, with support from the ESRC, the first UK qualitative data archiving project on a national scale was established at the University of Essex. It was set up as a small unit based in the Department of Sociology under the direction of a member of the department, Paul Thompson, an eminent oral historian. Its first task was a rescue operation aiming to seek out the most significant material created by research from past fifty years. The second was to work with the ESRC to implement a Datasets Policy (ESRC 2002) to ensure that for current and future projects the unnecessary waste of the past did not continue. Qualidata was not set up as an archive itself, but as a clearing house and an action unit, its role being to locate and evaluate research data, catalogue it, organize its transfer to suitable archives across the UK, publicize its existence to researchers and encourage re-use of the collections (Corti et al. 1995; Thompson and Corti 1998).

In the mid-1990s, the Qualidata unit pioneered systematic procedures for archiving and disseminating qualitative data within a meaningful international social 
science framework, rather than using purely historical archival practises. Traditional archival 'box' listings do not utilise any consistent terminology in identifying or describing the 'data' elements of a personal paper collection. Thus resource discovery across collections, such as a search for 'field notes from the 1970s in the area of health', is not possible. Data types certainly cannot be distinguished from an archival repository listing.

The procedures included: sorting, processing and listing both raw data/and accompanying documentation (metadata); systematically describing studies for webbased resource discovery systems; establishing appropriate ethical frameworks and mechanisms of access; and training in the re-use of qualitative data (Corti 2000, 2002). These were developed from working closely with traditional archivists and data archivists. A number of test data sets were used to establish which descriptive schema matched the needs of a data collection-based archive and what kinds of descriptive elements met users' needs.

By 2005, Qualidata has acquired, processed and catalogued over 140 datasets, and catalogued a further 150 already housed in archives across the UK. Surviving "classic studies" data from key researchers were also rescued, including well-known British projects such as Elizabeth Bott's study on Family and Social Network (1956); John Goldthorpe et al.'s The Affluent Worker (1968); Stanley Cohen's Folk Devils and Moral Panics (1971); the entire life's work of pioneering UK researchers such as Peter Townsend's Family Life of Old People (1957), The Last Refuge (1962) and Poverty in the UK (Townsend 1979); and Paul Thompson's life-history interview studies of The Edwardians (1975) and Families, Social Mobility and Ageing, an Intergenerational Approach (Thompson et al. 1990). Thompson and Corti (2004) provide an introduction to a selection of talks by some of these leading pioneers of UK social research given at a symposium in 2000. From 2001, Qualidata began a new life as a specialist unit housed within the UK Data Archive (UKDA) at the University of Essex, with a focus on acquiring and distributing digital data. The practical aspects of acquiring, preparing, providing access to and supporting users of digital qualitative data through such an archive set up are described later.

In the US, there is also a centre that has been systematically gathering qualitative as well as quantitative research data in order to make it available to other social science researchers. Founded in 1976, the Murray Research Center: a Center for the Study of Lives is a national repository for social and behavioural science data on human development and social change, with special emphasis on the lives of American women (James and Sorenson 2000). The archive holds more than 270 data sets with a wide range of topics, samples, and designs.

Finally, over the past few years there have been a number of other initiatives across the world that have sought to establish national archiving projects for qualitative research data. The national (mostly survey-based) Social Science Data Archives in Finland (Kuula 2000), Germany (Opitz and Mauer 2005), Switzerland (Eberle 2004), Denmark (Fink 2000), The Netherlands and Canada have been conducting preliminary investigations into extending the scope of their own collecting.

The accumulation of documented and available qualitative data resources has thus encouraged the take-up of secondary analysis. Greater re-use of data also reflects some of the efforts invested in promoting or re-packaging data collections to meet researchers', teachers' and students' needs. And, as resources grow and the promotional machines grind into action, so experiences of secondary research have

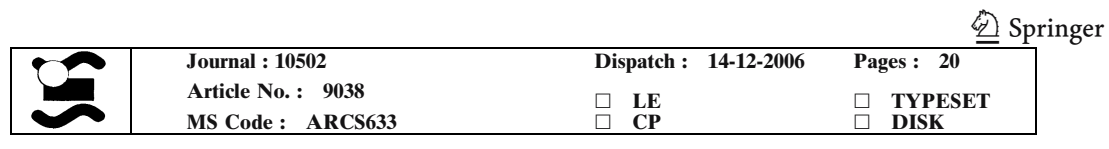


begun to find their place in social research literature, as the reflections in the FQS 212 Special Issue on Secondary Analysis of Qualitative Data testify (Corti et al. 2005a).

213 So, what can secondary analysis of qualitative data offer the researcher?

\section{The potential of data for secondary analysis}

215

216

217

218

219

220

221

222

223

224

225

226

227

228

229

230

231

232

233

234

235

236

237

238

Archived qualitative data exist to be re-used, revisited, reanalysed and compared with complementary data sources. There is a well-established tradition in social science of reanalysing quantitative data, although the skills base of statistically literate people continues to be under-developed. However, among qualitative researchers, to date, there has been no similar research culture that actively encourages new researchers or students in the social sciences to conduct reanalysis of data collected by other researchers. Until very recently there has been a distinct lack of public discussion of the issues involved and very little published "evidence" of the benefits and limitations of such an approach.

The re-use of qualitative data provides an opportunity to study the raw materials of recent or earlier research to gain both methodological and substantive insights, which are described later. Because new data are typically expensive to collect, using existing sources will save costs by avoiding duplication of research effort.

Corti and Thompson (2004) have already identified six approaches to re-using data which are described in relation both to theoretical issues raised more recently about re-analysis, and to the actual experiences of researchers. The authors expound the methodological, ethical and theoretical considerations relating to the secondary analysis of such qualitative data. Other contributions to the literature addressing these same issues have also begun to appear over the past few years (see Corti and Thompson 2004; Corti et al. 2004; Heaton 2004, Hammersley 1997; Corti 2000; Fielding and Fielding 2000; Thompson 1998; Szabo and Strang 1997).

The ways that qualitative data can be re-used are not dissimilar from those familiar for the secondary analysis of survey data. Indeed, the approaches have much in common with those familiar for the secondary analysis of survey data.

\section{Description}

240 The possibilities for using data descriptively are extensive-pictures of contemporary and historical attitudes and behaviour of individuals, groups and organisations, or societies can be gleaned. Indeed, significant data created now will in time become a potential historical resource. The oral testimonies of ordinary men and women can complement official and public sources such as newspapers and government reports, and such evidence can also be used to document individual lives from a biographical perspective, including those of significant researchers themselves. Sheridan (2000) notes how the material from the UK's Mass Observation has been used not only to provide historical evidence, but also to examine the role of the Mass Observation study itself in the social, political and cultural milieu of the 1930s and 1940s. Historical research methods thus become important here, and re-use of these materials will require the new investigator to first evaluate the evidence, examine its provenance, and assess the veracity of the sources. This may be a new practice for contemporary social researchers (Kynaston 2005). But original context is hard to

\begin{tabular}{|l|llll|}
\hline & Journal : 10502 & Dispatch : 14-12-2006 & Pages : 20 \\
& Article No. : 9038 & $\square$ LE & $\square$ TYPESET \\
MS Code : ARCS633 & $\square$ CP & $\square$ DISK \\
\hline
\end{tabular}


capture, and this is one of the major arguments voiced against re-use of others' data.

255 This point and attempts to remedy the problem is further elaborated later.

\section{Comparative research, restudy or follow-up study}

Qualitative data can be compared with other data sources or be used to provide comparison with other contexts, over other periods of time, and across other social groups and cultures. In Britain the original returns of the population census were kept as public records and have proved an invaluable basis for consultation in recent years. Sidney and Beatrice Webb (1894), on completing their pioneering study of British trade unionism, archived their field notes from their national sample of interviews, which still feature as the principal source of information on trade unionism in the late 19th century. Equally well known early classic restudies include Seebohm Rowntree's (1901) repeated surveys of poverty in York and Hubert Llewellyn Smith's (1930-1935) repeat of Charles Booth's (1891-1902) poverty survey in London. In anthropology a classic example is the controversial restudy and reinterpretation by Oscar Lewis (1963) of Robert Redfield's (1930) research on the village of Tepotzlan in Mexico. But the restudies made use of the published methods, not necessarily the raw paper data.

Comparison brings greater power to answer research questions, for example when a dataset can be combined with data beyond its own sample or geographical limitations. Equally, samples from original studies that have been preserved can be followed up, typically with the involvement of the original investigator, and sometimes with new ethical approval. An example is Glen Elder's Children of the Great Depression (1974), based on both new fieldwork and a reorganisation of the earlier interviews and participant observation of the Berkeley and Oakland cohorts interviewed on a regular basis since the 1920s, archived by the Murray Research Centre. Follow-up studies typically require approval from a research ethics committee, as it involves re-contacting original participants who may not have been expecting contact from new researchers. In addition, particularly in the health field, original investigators are often keen to become collaborators, rather than just being cited as the original data collectors (Corti and Wright 2002).

\section{Re-analysis or secondary analysis}

Reanalysing qualitative data allows both for re-interpretations and also for new questions to be asked of the data. Julie Charlesworth and Janet Fink (2001) draw upon original research data from Peter Townsend's study of institutional care published as The Last Refuge (Townsend 1957), to illustrate the potential which this archived data holds for the analysis of such topics related to workplace and organisational dynamics. Alternatively, new angles can be applied and new methods employed which may not have been possible at the time of the original data analysis. Sometimes new analytical tools can highlight parts of data that were previously ignored in the original analysis, offering the chance to revisit and reanalyze material, even if already written up (Akerström et al. 2004). Typically, the richer the original research material, the more potential there is for further exploitation.

Nigel Fielding and Jane Fielding (2000) revisited Stan Cohen and Laurie Taylor's (1972) original analysis of long-term imprisonment of men in maximum security published as Psychological Survival. Their restudy highlights the value of secondary

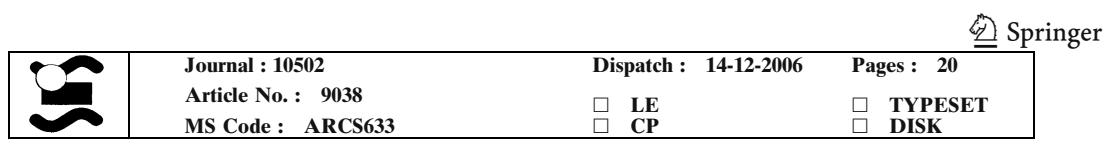


analysis in addressing sensitive topics or hard to reach populations, by extracting the maximum value from those studies which are able to negotiate access.

In the US, research using the Murray Research Centre data collection demonstrates the ways that qualitative data can be restructured for new research, for example creating new prospective studies out of existing ones and combining multiple data sets for multi-cohort designs. Jacqueline James and Anamette Sørensen (2000) discuss how the original transcripts of in-depth interviews, observations, and responses to tests, can be especially valuable in applying different perspectives and renewed scoring procedures to the original data.

Research design and methodological advancement

A study of the research methods of an original research investigation, such as the sampling methods, data collection and fieldwork strategies and interview guides of earlier research can help in the design of a new study or the development a methodology or research tool. Paul Thompson reflects on the role of drawing on existing interview guides designed by earlier researchers in a similar field (Savage 2005; Corti and Thompson 2004; Thompson 2000b).

There is a growing emphasis on publishing methodological details in reports, and books, but all too often the details offered are frustratingly brief and sanitised. One of ESDS Qualidata's roles, as we shall see later, is to try to encourage and devise better strategies for capturing the methodological perspectives and details under which studies are undertaken (across all stages) thereby providing greater context for a secondary user who may be unfamiliar with a set of raw data. Researchers' own research notes and fieldwork writings can offer much insight into the history and development of the research and also help inform new thinking.

\section{Verification}

Archived data can be scrutinized with scientific rigour to support or challenge a set of findings or to appraise the method. The practice of opening data for inspection is becoming increasingly important in the natural sciences, with the aim of encouraging more transparent research. We have also seen in the UK the recent start up of training in master classes on yerification in the field of quantitative economics, the Replication Workshop-Estimating Time-Series-Cross-Section Models with Comparative Political Economy Data (ESRC Oxford Spring School). This initiative is funded through the ESRC Research Methods Programme, which has a strong training component aiming to improve the standards of research methods across the UK social science (RMP Website 2005).

Martin Hammersley (1997) discusses the benefits and weaknesses of using "replication" to check findings, arguing that true scientific replication is not possible as studies generally do not have equal social phenomena. Restudies suffer from differences in time and the researchers' subjective perspectives, but welldocumented data sets can help the new investigator to reconstruct the evidence by re-trácing the original analytic steps. Hammersley (1997) and others correctly argue that replication is not an appropriate objective for secondary analysis, partly because of the problem of context. The loss of the holistic context of a study means that it is unlikely that the research process could ever be made fully explicit—-the path of qualitative analysis is never linear, and almost always involves a degree of trial and 
error in the pursuit of interesting lines of investigation. Retention of the original coding frames and analytic notes means that these can be reapplied by another investigator. Nigel and Jane Fielding (2000) further suggest that qualitative software may help the process of verification. We discuss the use of Computer Assisted Qualitative Data Analysis (CAQDAS) software (such as Atlas-ti or N-Vivo) in the teaching of secondary analysis later on in this paper.

\section{Teaching and learning}

351

352

353

354

355

356

357

358

359

360

361

362

363

364

365

366

367

368

369

370

\section{1}

372

373

374

375

376

377

378

379

380

381

382

383

384

385

The use of real-life data in teaching substantive or methodological perspectives in the social sciences adds interest and relevance to courses. Students who gain their experiences of data analysis from the use of archived data from published studies can gain a good understanding of the complexity of data analysis as it relates to the "real" world. Students gain the opportunity to understand the rationale for collecting data and can develop critical faculties to judge the strengths and weaknesses of a particular data collection strategy or analytic approach. Data can be chosen to be of particular relevance to the subject being taught and thus can bring both substantive and methodological topics alive.

Older 'classic' studies in the social sciences and more contemporary focused sets of transcripts along with supporting documentation can provide valuable material for social science teaching, both in research methods and in substantive areas. Students can learn many fundamental aspects of qualitative research, and the theoretical and methodological strategies that helped to create chosen datasets, while also gaining first-hand experience of critically re-analysing and comparing data from well-known sources. Learning about the work of researchers who have made a significant impact in their field allows young researchers to take the best practice elements from this work and further develop them in their own research work (c.f. Zeitlyn 2000). Examples of using qualitative data in teaching and learning are discussed by Corti and Bishop (2005).

\section{Difficulties in re-using data}

But the practice of secondary analysis of qualitative data is not a commonplace research activity. There appears to be different and perhaps more challenging intellectual, epistemological and practical problems for the user to consider compared to confronting numeric data, although re-use of any dataset collected by a third party can be beset with complexity. Why has there been a reluctance to draw on material created by other researchers? Is it that it is a problem of the implicit nature of qualitative data collection and analysis? Or is it a question of lack of time to get fully acquainted with research materials created by someone else? How constraining is informed consent? And what about scientific verification-is there insecurity about the exposure of one's own research practice? The recent, though sparse, literature points to a number of key concerns regarding the practice of re-using qualitative data.

However, in discussing the issues directly with qualitative researchers, it appears that the views are by no means homogenous. In fact, when asked what, if any, barriers existed to further exploitation of data by a secondary analyst, responses varied from overt support for sharing one's own data to vehement displeasure at the

\begin{tabular}{|l|llll|} 
& & & & \\
\hline
\end{tabular}


388

389

390

391

392

393

394

thought of being asked to share a 'possession' considered to be of personal value (Corti et al. 1995; Corti 2000; Fink 2000).

The key issues that present themselves as difficulties in both re-using and sharing data are 5-fold, as identified by Corti and Thompson (2004). These are ethical and consent considerations; representation, coverage and context of the research and fieldwork; unfamiliarity with the methods; lack of infrastructure for data-sharing; misinterpretation of data; threat to intellectual property rights. For a discussion on ethics, see Corti et al. (2000) and see the ESDS Qualidata web pages on this topic (ESDS Qualidata website 2004).

The architecture of data provision: traditional archive repository versus data archive

In the earlier debates as to how to operate the UK national qualitative archive, two models of data storage and provision were identified: a centralised facility in a single location or a hub and spokes model. These are of course extreme models representing opposite ends of the spectrum. The UK Qualidata was established using the latter approach, with the Centre as the hub, bearing responsibility for evaluating, acquiring, preparing, documenting, setting access conditions, transferring and publicising data. A network of traditional archives, largely situated in University libraries, acted as spokes which enabled the long-term storage of data. For Qualidata's initial stages, when most of the research data handled were paper-based, it was very clear that a distributed or 'clearing house' model had costs savings over a centralised one. All the long-term costs of maintaining this paper-based material have been off-loaded to the archives which have agreed to house the data, and ESRC has been saved the mounting expense of maintaining its own central archive with appropriate storage conditions, trained archival staff, maintaining facilities for research users, etc.

However, this era is almost over. We see now that there are scarcely any new datasets in the UK to archive, which are not available in electronic form, or do not already now sit in national archives. As knowledge about deposited sources of data increases, so do requests for help in finding and obtaining suitable datasets.

Should data reside in one place or be dispersed? The former can ensure standards-in terms of data quality, preservation and controlled access, whilst the latter places the emphasis on the distribution of material to a network of high-class traditional archives, many chosen because they are at centres of high research activity in particular fields. It may be that the ideal solution for the (predominantly digital) future can combine both models, with data stored both centrally and locally giving a double benefit to a host of dispersed and disparate user communities.

Descriptive systems also differ between the two communities. An archivist will typically catalogue a collection, say of a retired sociologist's papers by chronology, perhaps subdividing them into periods when the person held different professional roles. By contrast, a data archivist will identify and pull out the distinct research studies and catálogue them at the study level. This has implications for users tapping into finding aids. Typically, the user of qualitative data is a social scientist, rather than a historian, and thus study level description is critical because they wish to re-analyse or replicate a study, as discussed earlier. Because many empirical undertakings are now utilising mixed methods strategies in their research design, it is even more crucial to describe data at the study level to cover, for example, both

\begin{tabular}{|l|llll|}
\hline & Journal : 10502 & Dispatch : 14-12-2006 & Pages : 20 \\
Article No. : 9038 & MS Code : ARCS633 & $\square$ LE & $\square$ TYPESET \\
\hline
\end{tabular}


numerical datasets and qualitative interview materials. The traditional archive community uses the international cataloguing standard, the General International Standard Archival Description ISAD $(G)$ while the data archiving community use the Data Documentation Initiative (DDI) (ISAD(G) 2000; DDI 2005). While some of the descriptive elements map, they follow the different logic of the communities' 440 own practices: personal or corporate fonds typically by chronology versus unique 441 study or data description.

\section{UK National Qualitative Archive: ESDS Qualidata}

443 From 2001, the original Qualidata unit, mentioned earlier, began a new life as a 444 specialist unit housed within the UKDA at the University of Essex, with a focus on acquiring and distributing digital data. The key drivers behind merging the data services were multi-fold: the desire to create a one-stop social science data shop built around a single hub giving Essex a unique portfolio of data expertise and technological vision; the need to strengthen alliances to meet a tendering process ensuing from the ESRC's strategic review of their data archiving and dissemination services; the wish to streamline and simplify the data deposit process for ESRC depositors; and a growing need to reduce the demarcation between qualitative and quantitative data.

As Qualidata did not physically hold all the data it publicises in its catalogue, other than having a degree of access control over the local paper based archive at Essex (the National Social Policy and Social Change Archive collection), users are often put off by the fact that they may, for example, have to travel to Scotland to access a single dataset based in a Scottish Repository. Moreover, Qualidata previously found itself having to acquire data on the user's behalf, for example by arranging to get copies made for and dispatched to a user. In the short-term, there was no getting round users having to visit archives in person to access large paper-based collections, as repositories are in no position to digitise all their holdings.

Without the merger, the Qualidata service, which was set up as a small pilot service, would probably not have survived in the longer term. Phase I of the integration process was complete by October 2001, when many of the strategic and operational procedures for data acquisition, processing, metadata creation and dissemination were merged in to sit along side the handling of numeric data. Crossdivisional training of personnel of the merged organization was initiated to broaden data processing skills to cover a wider range of data types, including mixed methods datasets.

ESDS Qualidata is now a specialist service of the broader UK Economic and Social Data Service (ESDS) led by the UKDA at the University of Essex. The ESDS is a national data archiving and dissemination service that came into operation in January 2003. The service is a jointly funded initiative sponsored by the Economic and Social Research Council (ESRC) and the Joint Information Systems Committee (JISC) and provides access and support for an extensive range of key economic and social data, both quantitative and qualitative, spanning many disciplines and themes. The dedicated qualitative data service provides access and support for a range of social science qualitative datasets and is responsible for generating a number of data

\begin{tabular}{|l|lll|}
\hline & & & \multicolumn{2}{l}{ Springer } \\
& Journal : 10502 & Dispatch : 14-12-2006 & Pages : 20 \\
Article No. : 9038 & & $\square$ LE & 0 \\
MS Code : ARCS633 & CP & DISESET \\
\hline
\end{tabular}


480

481

482

483

484

485

486

487

488

489

490

491

492

493

494

495

496

497

498

499

500

501

502

503

504

505

506

507

508

509

510

511

512

513

514

515

516

517

518

enhancements, and for providing information and training resources that focus on strategies for re-analysing qualitative data.

The focus is on acquiring digital data collections from purely qualitative and mixed methods contemporary research from a wide range of social science disciplines and from UK-based "classic studies", which are typically post-war studies of British society. Data supported include: in-depth and semi-structured interviews; focus groups; field notes and observations; personal documents and photographs. All data are considered, either proactively or reactively, but the main inflow is via ESRC research grants, through which primary data are collected. Thus from an acquisitions point of view, the UKDA has been fortunate in that it has been sufficiently supported to build up both a viable operation and a stock of data through the framework and infrastructure of a national policy for archiving data. As such, this UK model, that is now, thankfully, beyond its probation phase, offers a pioneering exemplar to other countries as to how to enable the systematic collection and secondary use of qualitative data.

ESDS Qualidata plays a pivotal role in working closely with data creators to ensure that high quality and well-documented qualitative data that have longer-term value are produced. As part of its core functions, both general guidance and a dedicated advisory service are provided for data creators and depositors on research project management, issues of confidentiality and consent, and documentation of data for archiving. Taken seriously at the start of a research project, good practice across these areas extends the usability lifetime of data and potentially enables creative and flexible re-use of data.

ESDS Qualidata, and indeed, the Murray Research Center, have acquisitions policies to ensure that all materials deposited meet certain criteria: that data are documented to a minimum standard, are in appropriate formats, are complete, and that confidentiality, data protection and copyright issues have been addressed. Priorities must also be assigned to data, so that the inflow of data meets the resources available for processing. Potential studies are thus always evaluated from a longrange perspective to predict their future value. Priorities focus on:

- the historical value of the study

- data complementary to existing data holdings

- data that have further analytic potential than the original investigation, i.e. have not been exhaustively analyzed

- data based on large-scale national samples

- data which are longitudinal in design

- the possibility of further follow-up of the sample

- mixed methods data

- studies that include a wide range of measures

Finally, ESDS Qualidata offers a resource discovery hub via the UKDA online catalogue that holds some 4000 data collections across the disciplinary and methodological spectrum. The catalogue also points to other accessible sources of qualitative data across the UK not physically held by the UKDA. The service continues its earlier role in facilitating the preservation of important large paper qualitative research collections (for deposit in traditional paper archives), top level cataloguing and, where appropriate, digitizing samples of these collections. 

and online data access

528

529

530

531

532

Users of qualitative data want instant access to data and they want more than just one collection. But simply preserving and disseminating the original research documents is not enough. Enhancing qualitative data is one of the keys to increasing visibility and enabling easier and more effective use by researchers and teachers. This does not mean changing raw data in any way it simple means adding value to data by providing enhanced resource discovery and richer comprehension about the data and its provenance.

In the context of archiving qualitative data, for ESDS Qualidata, enhancement has three meanings. The first consists of digitising by converting paper to some electronic form. Many of the most valuable collections (e.g., classic sociology studies) exist only in paper format and require this type of enhancement to become web-enabled. Currently, data are digitised to three different levels: searchable PDF; digitised for download; and XML-tagged for online access.

The second form of enhancement of contextual material involves augmenting a data collection with additional materials to make the collection more useful to potential researchers. One of the key barriers cited by those who do not support the archiving of qualitative data, other than ethical constraints, is the implicit nature of qualitative data collection and analysis, the 'problem' of not having access to original context and reflexivity found in the original fieldwork and data analysis. How can new researchers fully engage with research materials created by someone else?

ESDS Qualidata has always argued that some high quality data is better than no data for the community, and take the position that the value of raw data can be enhanced by providing as much context as possible-provided by the original researcher or by researching more about their work. The enhancement of context involves augmenting a data collection with additional contextual materials to make the collection more useful to potential researchers, for example by adding materials that reveal both the context and the process of the original research, and depends on the nature of the collection, the complexity of the methodology and the materials available. Any content that enriches context or explains in detail how the original research was actually done is extremely valuable to researchers embarking on secondary analysis, but the extent of this enhancement varies greatly as it depends both on the nature of the collection (complexity of the methodology, for example) and on what materials are available from depositors of the research. These enhanced user guides may include samplers that provide highlights of key qualitative materials to illustrate the potential of the collection for research and teaching. Typically, the materials are assembled into a user guide and made available in bookmarked PDF for download via the catalogue with the data.

As an example, a classic sociology collection, Mothers and Daughters: Accounts of Health in the Grandmother Generation, 1945-1978 (SN 4943) by the well known UKbased sociologist, Mildred Blaxter was released. The preparation involved: conversion of data from paper to searchable Word and RTF format by OCR, involving extensive editing and formatting of 46 interview transcripts, production of a brief Scots dialect glossary, and the compilation of extracts from an interview with the author about the experience of conducting this research.

\begin{tabular}{|l|llll|} 
& & & & \\
\hline
\end{tabular}


The third is about providing online search and browse facilities to access raw data using a web browser. The original pilot Edwardians Online has been expanded to form the generic ESDS Qualidata Online system (www.esds.ac.uk/qualidata) centred on a vision for more flexible access to digital qualitative data via real-time online browsing of data and utilising non-proprietary XML-based formats and systems for preserving, searching, and disseminating qualitative data (Corti and Barker 2003). The system supports more powerful resource discovery and offers greater scope for searching and browsing content of data (over higher level study-related metadata). Since users can search and explore (textual) content across different datasets directly, data can be retrieved immediately. The advantages are that a system based on common standards (based on XML) provides access to qualitative data via a common interface using a standard web browser. In this system, depending on the dataset, various combinations of interview transcripts, interview summaries, methodology and background materials across multiple datasets are available to browse and search. For example, researchers can now select and search interviews from multiple datasets, including Mothers and Daughters, and Paul Thompson's more recent study of 100 Families: Families, Social Mobility and Ageing, an Intergenerational Approach (Thompson et al. 1990). XML mark-up allows potential linking to other various research sources of data, such as that envisaged by e-social science thinking (Muhr 2000).

The format and mark-up of data also determine the usefulness of a collection. There is a debate about the long-term value of coded data-that created in the original analysis phase-mainly because the coding process is subjective, often geared towards specific themes, and therefore may not be applicable to the secondary analyst's topic of investigation. For larger studies, however, there is a stronger case for retaining the principal researcher's coded data, in order to aid searching within voluminous bodies of text. Indeed, the Edwardians Collection in the ESDS Qualidata Online system incorporated the structure of the existing coding to provide navigation through the huge bulk of text—some 50,000 pages of interview transcript.

\section{Audio-visual materials}

604 Since audio-visual materials are increasingly being created in the course of quali605 tative research, an archive needs to consider housing and providing access to them. 606 More recently, ESDS Qualidata has begun to develop in-house methods for pro607 cessing audio interviews that includes digitisation. While the service does not handle 608 many audio-visual collections, mainly due to confidentiality issues, it is expected that 609 more researchers will be utilising digital recorders with consent obtained to archive 610 data. As such, ESDS will be looking in future to make these available on-line as part 611 of the collection.

\section{Publicity and outreach}

613 Given that, to some extent, the culture of secondary analysis of qualitative data 614 materials is still emerging, an important remit for a unit supporting qualitative data 615 is to raise the level of awareness of the availability of, and potential for, utilising

\begin{tabular}{|l|llll|}
\hline & Journal : 10502 & Dispatch : 14-12-2006 & Pages : 20 \\
& Article No. : 9038 & $\square$ LE & $\square$ TYPESET \\
MS Code : ARCS633 & $\square$ CP & $\square$ DISK \\
\hline
\end{tabular}


616 qualitative data sources in research, learning and teaching. Many researchers are not 617 sufficiently informed about possible methods and technical means for archiving and 618 secondary analysis, including issues of anonymisation, confidentiality and ethics as well as to valid, creative and resource saving ways of how to ask 'new questions from the old data'. Thus promotional efforts to draw researchers and teachers attention to the possibilities of re-using data sources are of great importance. A wide range of dissemination and outreach activities undertaken by ESDS Qualidata provide support for, and awareness of, the potential of qualitative data. Provision of a dedicated help desk facility, regularly updated web pages and FAQs, and an email discussion list as a forum to host debates on issues arising in the use of qualitative data are productive ways of helping maintain a user community.

The most important promotional media is the web, key newsletters and also journals. Web content should include: regularly updated data, development, news and events web pages; a section on re-using data; and detailed but jargon-free guidance on creating and depositing data. ESDS Qualidata's web pages on re-use provides: an overview of ways of re-using data; an FAQ on re-use; and is regularly updated, with a bibliography of articles addressing re-use and case studies of re-use including reflections and commentary. ESDS Qualidata contributes to the regular ESDS Newsletter, UKDatabytes which highlights new data collections, recent developments in data services and training opportunities.

The ESDS Qualidata team also publish in other newsletters and journals where time permits on various aspects of archiving, accessing and re-using qualitative data. Although academic output is not required of ESDS staff, it is always desirable and is nevertheless considered to be valuable in helping with the evangelist mission of building new communities of practice. Finally, it is also useful to make an appearance in newsletters and journals which will reach teachers and student populations.

A programme of training events and activities is also critical to expanding the user base. ESDS Qualidata runs a series of workshops that aims to enhance the methodological and substantive understanding, and secondary analytical potential, of archived qualitative data sources. These include data creation workshops-how to make data shareable; awareness days and road shows; tailored user and "data confrontation" workshops; thematic events, by discipline or method; secondary analysis of existing sources; exploration of data sources and data browsing systems; and using CAQDAS software to explore shared data.

CAQDAS software is increasingly used by qualitative researchers to help manage large amounts of primary data-interview materials, images, photos, diagrams and even video and audio recordings. Added to this are notes, memos, comments and coding that are created during the analytic process. Coding is a key tool for keeping a record of analytic thoughts about the data and a way in which researchers develop an analytical understanding and interpretation of their data. Keeping most of the information in CAQDAS software and using the organising features of the packages help keep it organised. The software supports the interactive coding of text and has search and retrieve tools, and some enable modelling, building networks and linkages across objects. However many researchers still use paper and scissors methods to classify and organise their data, typically because they are not technically proficient. It is thus beneficial to help promote digital resource and computer software together.

Workshop sessions on data preparation and management have also been 664 invited from various ESRC Research Programmes, the International Sociological

\begin{tabular}{|l|llll|} 
& & & & \\
Journal : 10502 & Dispatch : & 14-12-2006 & Pages : 20 \\
Article No. : 9038 & $\square$ & LE & $\square$ TYPESET \\
MS Code : ARCS633 & $\square$ & CP & $\square$ DISK \\
\hline
\end{tabular}


665 Association (ISA) and the International Association for Social Science Information 666 Services and Technology (IASSIST) conference committee. It is vital that archives 667 holding qualitative data form part of these communities and keep up to date with the 668 developments and trends in the field.

Training events are always fully booked, suggesting that the supply cannot even begin to meet the demand in the qualitative domain. Online guides to help get users started are also useful ways to complement face-to-face training resources. A good way of promoting these resources and hence methods of secondary analysis is to encourage teachers to participate in evaluating training resources.

\section{So, who is using data?}

ESDS Qualidata has witnessed an important increase in the numbers accessing qualitative data, with a total of 56 requests for datasets recorded during the year 2003-2004, compared with an average of around 20 in previous years. This reverses the trend of previous years where most substantial usage was through access to the 680 paper classic sociology collections that ESDS Qualidata had archived in the past. 681 As hoped, core usage is now through the central UKDA/ESDS catalogue showing 682 that the hard work put into processing this material is paying off. One final 683 interesting fact is a breaking of the cycle where most researchers were making use 684 of only one or two key collections and overlooking recently-released material. More and more orders are being placed for the recent data. The user figures do not compare with survey data as there are literally thousands of survey data collections in the UK Data Archive's catalogue (about 3500 to some 70 qualitative), but as an indication of contrast, some 17,800 survey datasets were accessed in the same year.

Evidence from ESDS Qualidata suggests that older "classic" studies in the social sciences can provide extremely valuable material for new research and for social science teaching, both in research methods and in substantive areas. The demand for the classic studies has been significant, demonstrated by the top four requested datasets:

- SN 2000 Family Life and Work Experience Before 1918, 1870-1973Paul Thompson;

- SN 4723 Family Life of Old People, 1865-1955-Peter Townsend;

- SN 4871 Affluent Worker, 1961-1962-Frank Becchofer et al.;

- SN 5072 Mothers Alone, 1955-1966-Mildred Blaxter.

Creating and delivering more visible and packaged online electronic resources is a key way to facilitate both the usage of data and training in methodological skills among younger researchers and students. In order for these products to be of most benefit, they need to be accompanied by substantive and methodological commentary on the project and data, hands-on exercises, the availability of face-to-face training, and finally continuing individual support. Bespoke sets of interviews have been prepared on demand for teachers on a variety of courses: introductions to CAQDAS packages; oral history; discourse analysis; and general research methods courses.

\begin{tabular}{|l|llll|}
\hline & Journal : 10502 & Dispatch : 14-12-2006 & Pages : 20 \\
& Article No. : 9038 & $\square \quad$ LE & $\square$ & TYPESET \\
MS Code : ARCS633 & $\square$ CP & $\square$ DISK \\
\hline
\end{tabular}




\section{Conclusion}

710

711

712

713

714

715

716

717

718

719

720

721

722

723

724

725

726

727

728

729

730

731

732

733

734

735

736

737

738

739

740

741

742

743

744

745

746

747

The first part of this paper demonstrated how existing sources of qualitative data can be re-used. Firstly, this is because secondary analysis makes more effective use of material which is costly to collect; secondly, it enables further exploration of the data from a new perspective; thirdly, it enables comparative research to be carried out in a number of contexts (e.g. geographically, over time, cross-culturally); and last, it allows for verification of the original study. In many ways these methods parallel those that are used and documented for the secondary analysis of survey data: comparative research, replication or restudy; asking new questions of old data; the strengthening of scientific inquiry through the open discussion of methods; help in new research designs; and providing resources for training in research and substantive learning.

There are important gains to be made from re-analysis. At the start of a research project, it can be invaluable in providing a sense of the topics which can be successfully covered in interviewing, and therefore make the pilot stage of the new project both more effective and also much swifter. At a later stage a comparable interview set may also provide a crucial wider sample base for testing the interpretations which are emerging. Finally, by making research data available to re-analysis by others, the investigator may multiply the outcomes from this initial research through the publications of others from the same material. Equally there are methodological and practical difficulties in re-using data, which include understanding the coverage and context of the research; ethical and consent considerations; unfamiliarity with the method; and the general lack of suitable data available.

Over the last 5 years we have witnessed a new culture of the secondary use of qualitative data, which has been largely borne out of the UK data-sharing policy. It is unfortunate that there is no evidence of similar research provision or research cultures in other countries on this national scale, but it is highly likely that this will change over the next decade.

This emerging research culture needs to be nurtured by acquiring relevant data and documenting and presenting it in user-friendly ways that focus on quick and easy digital access. Qualitative data services can help fulfil this role by encouraging a culture of sharing in research practice and enabling support; developing appropriate collection priorities, creating digital resources for teaching and research, and by offering support and outreach activities such as training. It is also significant from the experiences of well established but very focussed archives-the Mass Observation Archive in Britain and the Murray Research Center in the US - that a particularly effective model is to combine archiving with in-house research on the archived collections held: this generates both relevant acquisitions and a high level of use by researchers. Finally, looking into the future, innovative on-line data access and analysis tools are very likely to both encourage and facilitate the re-use of qualitative data.

\section{References}

Akerström M, Jacobsson K, Wästerfors D (2004) Reanalysis of previously collected material. In: Seale C, Gobo G, Gubrium JF, Silverman D (eds) Qualitative research practice. Sage, London, pp 344-358

Booth C (1891-1902) Life and labour of the people in London. Williams and Norgate; Macmillan, London

Springer

\begin{tabular}{|l|llll|}
\hline & Journal : 10502 & Dispatch : 14-12-2006 & Pages : 20 \\
Article No. : 9038 & MS Code : ARCS633 & $\square$ LE & $\square$ TYPESET \\
\hline
\end{tabular}


Bott E (1956) Family and social network. Tavistock, London

Charlesworth J, Fink J (2001) Historians and social science research data: the Peter Townsend Collection. History Workshop J 51:206-219

Cohen S (1971) Folk devils and moral panics. The creation of the mods and rockers. Basil Blackwell, Oxford

Cohen S, Taylor L (1972) Psychological survival: the effects of long-term imprisonment. Allen Lane, London

Corti L (2000) Progress and problems of preserving and providing access to qualitative data for social research - the international picture of an emerging culture. Forum Qualitative Sozialforschung/Forum Qual Soc Res 1(3), Art. 2. http://www.qualitative-research.net/fqs-texte/3-00/300corti-e.htm. Cited: 11 Jan 2004

Corti L (2002) Qualitative data processing guidelines. Qualidata, UK Data Archive, University of Essex, Colchester

Corti L (2003) Qualitative data archiving. In: Lewis-Beck M, Bryman A, Futing Liao T (eds) The sage encyclopaedia of social science research methods, vol 1. Sage, London, pp 146-148

Corti L (2004) User support. In: Bergman M, Eberle T (eds) Qualitative inquiry: research, archiving and re-use. Swiss Academy of Humanities and Social Sciences, Bern, pp 153-167

Corti L, Barker E (2003) Edwardians online: An XML application for qualitative data. ASSIGnation 12(2):29-33

Corti L, Bishop L (2005) Strategies in teaching secondary analysis of qualitative data. Forum Qualitative Sozialforschung/Forum Qual Soc Res 6(1). http://www.qualitative-research.net/fqstexte/1-05/05-1-47-e.htm. Cited 31 Jan 2005

Corti L, Day A, Backhouse G (2000) Confidentiality and informed consent: Issues for consideration in the preservation of and provision of access to qualitative data archives. Forum Qualitative Sozialforschung/Forum Qual Soc Res 1(3), Art. 7. http://www.qualitative-research.net/fqs-texte/ 3-00/3-00cortietal-e.htm. Cited: 11 Jan 2004

Corti L, Foster J, Thompson P (1995) Archiving qualitative research data. Social Research Update 10, Department of Sociology, University of Surrey

Corti L, Thompson P (2004) Secondary analysis of archive data. In: Seale C, Gobo G, Gubrium JF, Silverman D (eds) Qualitative research practice. Sage, London, pp 327-343

Corti L, Thompson P, Fink J (2004) Preserving, sharing and re-using data from qualitative research. In: Cassell C, Symon G (eds) Essential guide to qualitative methods in organizational research. Sage, London, pp 288-301

Corti L, Witzel A, Bishop L (eds) (2005a) Secondary analysis of qualitative data. Forum Qualitative Sozialforschung/Forum Qual Soc Res 6(1). http://www.qualitative-research.net/fqs/fqs-e/inhalt1-05e.htm . Cited 31 Jan 2005

Corti L, Witzel A, Bishop L (2005b) On the potentials and problems of secondary analysis. An introduction to the FQS special issue on secondary analysis of qualitative data. Forum Qualitative Sozialforschung/Forum Qual Soc Res 6(1), Art. 49. http://www.qualitative-research.net/ fqs-texte/1-05/05-1-49-e.htm . Cited 31 Jan 2005

Corti L, Wright M (2002) Consultants' report to the Medical Research Council on the MRC Population Data Archiving and Access Project. UK Data Archive, University of Essex, Colchester

Council of European Social Science Data Archive (CESSDA). www.cessda.org.uk, IASSIST

DDI (2005) Data Documentation Initiative, Website. www.icpsr.umich.edu/DDI/

Eberle T (2004) Introduction. In: Manfred B, Thomas E (eds) Qualitative inquiry: research, archiving and re-use. Swiss Academy of Humanities and Social Sciences, Bern, pp 153-167

Elder G (1974) Children of the great depression: social change in life experience. University of Chicago Press, Chicago

ESDS (2004) ESDS Qualidata. http://www.esds.ac.uk/qualidata/. Cited 05 Nov 2006

ESRC (2002) ESRC datasets policy. ESRC, Swindon

ESRC (1999) Guidelines on copyright and confidentiality: legal issues for social science researchers. ESRC, Swindon

Fielding N, Fielding J (2000) Resistance and adaptation to criminal identity: using secondary analysis to evaluate classic studies of crime and deviance. Sociology 34(4):671-689

Fink AS (2000) The role of the researcher in the qualitative research process. A potential barrier to archiving qualitative data. Forum Qualitative Sozialforschung/Forum Qual Soc Res 1(3). http:// www.qualitative-research.net/fqs-texte/3-00/3-00fink-e.htm. Cited 05 Nov 2006

Goldthorpe J, Lockwood D, Bechhofer F, Platt J (1968) The affluent worker: industrial attitudes and behaviour. Cambridge University Press, Cambridge

\begin{tabular}{|l|llll|}
\hline & Journal : 10502 & Dispatch : & 14-12-2006 & Pages : 20 \\
Article No. : 9038 & $\square$ & LE & $\square$ TYPESET \\
MS Code : ARCS633 & $\square$ & CP & $\square$ DISK \\
\hline
\end{tabular}


814

815

816

817

818

819

820

821

822
Hammersley M (1997) Qualitative data archiving: some reflections on its prospects and problems. Sociology 31(1):131-42

Heaton Janet (2004) Reworking qualitative data. Sage, London

ISAD $(G)$ : General international standard archival description (2000) International Council on Archives. http://www.icacds.org.uk/eng/standards.htm. Cited 05 Nov 2006

James J, Sørensen A (2000) Archiving longitudinal data for future research. Why qualitative data add to a study's usefulness. Forum Qualitative Sozialforschung/Forum Qual Soc Res 1(3), Art. 23. http://www.qualitative-research.net/fqs-texte/3-00/3-00jamessorensen-e.htm. Cited 31, Jan 2004

Kuula A (2000) Making qualitative research material reusable: case in Finland. Forum Qual Soc Res 1(3). http://www.qualitative-research.net/fqs-texte/3-00/3-00kuula-e.htm. Cited 04 Nov 2006

Kynaston, D (2005) The uses of sociology for real-time history. Forum Qualitative Sozialforschung/ Forum: Qual Soc Res 6(1), Art. 31. http://www.qualitative-research.net/fqs-texte/1-05/05-1-45-e.htm. Cited 04 Nov 2006

Lewis O (1963) Tepotzlan restudied: life in a Mexican village. University of Illinois Press, Urbana Llewellyn Smith H (1930-1935) The new survey of London life and labour. P. S. King, London

Mruck K, Corti L, Kluge S, Opitz D (2000) About this issue. Forum Qualitative Sozialforschung/ Forum Qual Soc Res 1(3), Art. 1. http://www.qualitative-research.net/fqs-texte/3-00/3-00hrsg1e.htm. Cited 11 Jan 2004

Muhr T (2000) Increasing the reusability of qualitative data with XML. Forum Qual Soc Res 1(3). http://www.qualitative-research.net/fqs-texte/3-00/3-00muhr-e.htm. Cited 04 Nov 2006

Opitz D, Mauer R (2005) Experiences with secondary use of qualitative data-First results of a survey carried out in the context of a feasibility study concerning archiving and secondary use of interview data. Forum Qualitative Sozialforschung/Forum Qual Soc Res 6(1), Art. 43. http:// www.qualitative-research.net/fqs-texte/1-05/05-1-43-d.htm. Cited 12 April 2004

Redfield R (1930) Tepotzlan: a Mexican village; a study of folklife. University of Chicago Press, Chicago

Rex J, Moore R (1967) Race, community and conflict: a study of Sparkbrook. Oxford University Press, Oxford

RMP Website: Research Methods Programme (RMP) (2005) www.ccsr.ac.uk/methods/. Cited 05 Nov 2006

Rowntree S (1901) Poverty. A study of town life. Macmillan, London

Savage M (2005) Revisiting classic qualitative studies. Forum Qualitative Sozialforschung/Forum Qual Soc Res 6(1), Art. 31. http://www.qualitative-research.net/fqs-texte/1-05/05-1-31-e.htm. Cited 31 Jan 2005

Sheridan D (2000) Reviewing mass-observation: The archive and its researchers thirty years on. Forum Qualitative Sozialforschung/Forum Qual Soc Res 1(3), Art. 26. http://www.qualitativeresearch.net/fqs-texte/3-00/3-00sheridan-e.htm. Cited 31 Jan 2005

Stacey M (1974) The myth of community studies. In: Bell C, Newby H (eds) The sociology of community: a selection of readings. Frank Cass, London, pp 13-26

Szabo V, Strang V (1997) Secondary analysis of qualitative data. Adv Nurs Sci 20(2):66-74

Thompson P (1975) The Edwardians: the remaking of British society. Weidenfeld and Nicolson, London

Thompson P (1991) Pilot study of archiving qualitative data: Report to ESRC, Department of Sociology, University of Essex

Thompson P (1998) Sharing and reshaping life stories. In: Chamberlain M, Thompson P (eds) Narrative and genre. Routledge, London, pp 167-181

Thompson P (2000a) The voice of the past, 3rd rev edn; 1st edn 1978. Oxford University Press, Oxford

Thompson P (2000b) Experiences of re-analysing data in qualitative research. Forum Qualitative Sozialforschung /Forum Qual Soc Res [On-line Journal], 1(3), Art. 27. http://www.qualitativeresearch.net/fqs-texte/3-00/3-00thompson-e.htm. Cited 31 Jan 2005

Thompson P (2003) Interview with Mildred Blaxter. University of Essex, Colchester

Thompson P, Corti L (eds) (2004) Special issue: Celebrating classic sociology: pioneers of contemporary British qualitative research. Int J Soc Res Methodol Theor Pract 7(1)

Thompson P, Corti L (1998) Are you sitting on your qualitative data? Qualidata's mission. Soc Res Methodol Theor Pract 1(1):85-90

Thompson P, Itzin C, Abendstern M (1990) I don't feel old: the experience of ageing. Oxford University Press, Oxford

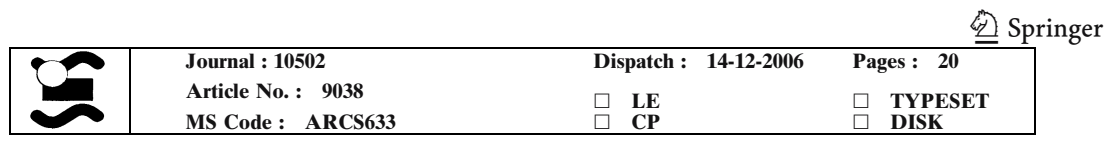


873 Townsend Peter (1957) The family life of old people. Routledge, London

874 Townsend P (1962) The last refuge, a survey of residential institutions and homes for the aged in $875 \quad$ England and Wales. Routledge, London

876 Townsend P (1979) Poverty in the United Kingdom, a survey of household resources and standards

877 of living. Penguin Books, London

Webb S, Webb B (1920) History of trade unionism, 1st edn 1894. Longmans, Green, London

UK Data Archive (2002a) UK data archive preservation policy. UK Data Archive, University of Essex

UK Data Archive (2002b) Cataloguing and indexing guidelines. UK Data Archive, University of Essex

Zeitlyn D (2000) Archiving anthropology. Forum Qualitative Sozialforschung/Forum Qual Soc Res 1(3), Art. 17. http://www.qualitative-research.net/fqs-texte/3-00/3-00zeitlyn-e.htm. Cited 31 Jan 2005

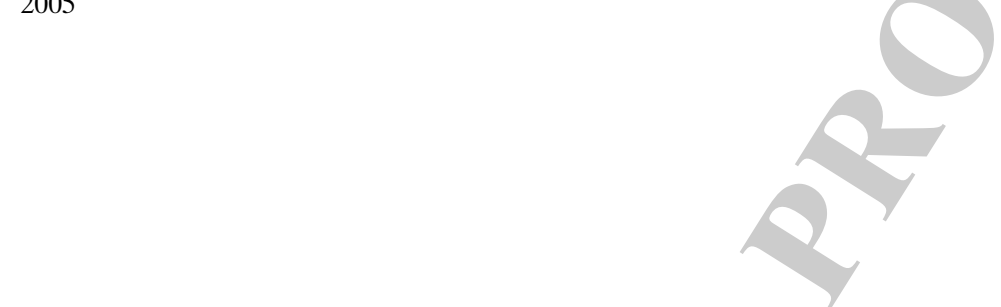

